\title{
Sialic acid mediated transcriptional modulation of a highly conserved sialometabolism gene cluster in Haemophilus influenzae and its effect on virulence
}

\author{
Gaynor A Jenkins', Marisol Figueira², Gaurav A Kumar', Wendy A Sweetman', Katherine Makepeace', \\ Stephen I Pelton², Richard Moxon', Derek W Hood ${ }^{1 *}$
}

\begin{abstract}
Background: Sialic acid has been shown to be a major virulence determinant in the pathogenesis of otitis media caused by the bacterium Haemophilus influenzae. This study aimed to characterise the expression of genes required for the metabolism of sialic acid and to investigate the role of these genes in virulence.

Results: Using qRT-PCR, we observed decreased transcriptional activity of genes within a cluster that are required for uptake and catabolism of 5-acetyl neuraminic acid (Neu5Ac), when bacteria were cultured in the presence of the sugar. We show that these uptake and catabolic genes, including a sialic acid regulatory gene (siaR), are highly conserved in the $\mathrm{H}$. influenzae natural population. Mutant strains were constructed for seven of the nine genes and their influence upon LPS sialylation and resistance of the bacteria to the killing effect of normal human serum were assessed. Mutations in the Neu5Ac uptake (TRAP transporter) genes decreased virulence in the chinchilla model of otitis media, but the attenuation was strain dependent. In contrast, mutations in catabolism genes and genes regulating sialic acid metabolism (siaR and crp) did not attenuate virulence.
\end{abstract}

Conclusion: The commensal and pathogenic behaviour of $\mathrm{H}$. influenzae involves LPS sialylation that can be influenced by a complex regulatory interplay of sialometabolism genes.

\section{Background}

Sialic acid (5-Acetylneuraminic acid, Neu5Ac) is a common sugar found as a terminal residue on glycoconjugates in many animals. In man, cell surface sialylation with Neu5Ac serves as a ligand for cell-cell adhesion, prevents complement activation and can help regulate tissue function and some cell signalling processes [1]. For Haemophilus influenzae, a Gram-negative bacterium found only in humans, the major surface glycolipid, lipopolysaccharide (LPS), can also be sialylated. This bacterium is an obligate commensal of the human respiratory tract but is able to cause significant disease. The majority of strains lack a capsule, so called non-typeable (NTHi) strains, and commonly cause otitis media $(\mathrm{OM})$, sinusitis and lower

\footnotetext{
* Correspondence: derek.hood@paediatrics.ox.ac.uk

'Molecular Infectious Diseases Group, University of Oxford Department of Paediatrics, Weatherall Institute of Molecular Medicine, John Radcliffe Hospital, Headington, Oxford, OX3 9DS, UK
}

respiratory tract infections, and occasionally invasive disease. NTHi LPS plays a role in the complex interactions with the host required in both its commensal and pathogenic behaviours. Sialylation of LPS is a relatively common structural modification among mucosal pathogens such as $H$. influenzae, with a reported role in virulence in a number of organisms. LPS sialylation influences the resistance of $H$. influenzae to the killing effects of normal human serum as evidenced by decreased survival in normal human serum of sialylation-deficient mutants, for example those in which the CMP-Neu5Ac synthetase gene (siaB) has been disrupted [2]. Moreover, the in vivo role of Neu5Ac as a critical virulence factor in the pathogenesis of experimental OM has been demonstrated as Neu5Acdeficient mutants were profoundly attenuated in animal models [3,4]. Sialylation of LPS interferes with the binding and activation of complement components of the host immune system on the bacterial surface [5]. Further, a role 
for LPS sialylation in 'biofilm' formation has been proposed that may be relevant to both the commensal behaviour and virulence of NTHi $[4,6,7]$.

$H$. influenzae cannot synthesize Neu5Ac de novo [8] and, in vivo, NTHi scavenges Neu5Ac from the host [3]. Neu5Ac is thought to be present at levels of about $0.5 \mathrm{mg} /$ $\mathrm{ml}$ in human serum [8] and in addition to being incorporated into LPS, Neu5Ac may also be used as a carbon and energy source [9]. Bioinformatic analysis has shown that the key genes required for the dissimulation of Neu5Ac are present in $H$. influenzae [8] and recent studies have identified a high affinity TRAP (Tripartite ATP independent Periplasmic) transport system encoded by the genes siaP and siaQM as the main uptake system of NTHi for procuring Neu5Ac [10,11]. The genes for sialic acid catabolism and procurement are contiguous on the $H$. influenzae genome $[8,12]$ and are arranged as two divergently transcribed operons (Figure 1). These nine genes are referred to as the sialometabolism gene cluster. The mechanism for regulation of $H$. influenzae sialic acid utilisation, whereby the entry of Neu5Ac into the catabolic pathway and incorporation in LPS is coordinated, is complex [12]. Located within the catabolic genes is siaR, encoding a protein containing two domains (helixturn-helix and sugar isomerase) associated with sugar metabolism and regulation [13,14], that acts as a repressor of sialometabolism genes [12]. cAMP receptor protein (CRP) has also been shown to regulate the expression of the sialic acid uptake but not the catabolic genes [12].

In the present study we used reverse transcriptase PCR to investigate sialometabolism gene transcription in $H$. influenzae wild type and sialometabolism mutant strains following growth of bacteria in the presence or absence of added sialic acid. Strains mutated in sialometabolism genes have been investigated in in vitro and in vivo assays and a complex process of regulation of Neu5Ac metabolism has been confirmed.

\section{Methods}

\section{Strains and culture conditions}

H. influenzae strain RM118 (Rd) is a capsule deficient derivative from a serotype $d$ strain for which the complete genome sequence has been obtained [15]. NTHi isolates used in this study are representative of the genetic diversity of $H$. influenzae [16], and have been reported previously [17]. H. influenzae was grown at $37^{\circ} \mathrm{C}$ in brain heart infusion (BHI) broth supplemented with $10 \mu \mathrm{g}$ haemin $\mathrm{ml}^{-1}$ and $2 \mu \mathrm{g}$ NAD ml${ }^{-1}$. BHI plates were prepared with $1 \%$ agar and supplemented with $10 \%$ (v/v) Levinthals base. For selection following transformation, $10 \mu \mathrm{g}$ kanamycin $\mathrm{ml}^{-1}$ was added to the medium. For some experimental growth of $H$. influenzae we used chemically defined medium (CDM) [18]. When appropriate, Neu5Ac was added at $25 \mu \mathrm{g} \mathrm{ml}^{-1}$ (BHI) or $30 \mu \mathrm{g} \mathrm{ml}$ (CDM) to the medium. Escherichia coli strain DH5 $\alpha$ was used to propagate plasmids and was grown at $37^{\circ} \mathrm{C}$ in $\mathrm{LB}$ broth [19] supplemented when appropriate with $100 \mu \mathrm{g}$ ampicillin $\mathrm{ml}^{-1}$ or $50 \mu \mathrm{g}$ kanamycin $\mathrm{ml}^{-1}$.

\section{Construction of $\boldsymbol{H}$. influenzae mutant strains}

The cloning and inactivation of siaP (HI0146), siaQ/M (HI0147) and HIO148 have been previously described [10]. Mutations were engineered in genes (HIO142HIO145) and in crp by the following general method; the gene of interest was first amplified by PCR using locus specific primers (listed in Table 1) and strain Rd chromosomal DNA as the template under conditions described previously [20]. Amplification products were ligated into PCR cloning vectors pT7Blue (Novagen) or pTOPO (Invitrogen) and transformed into E. coli. Correct clones were confirmed by restriction analysis and PCR amplification. Genes were inactivated by ligating the kanamycin resistance cassette $\left(\mathrm{kan}^{\mathrm{R}}\right)$, from pUC4Kan, into suitable restriction sites within the reading frame. $\operatorname{kan}^{\mathrm{R}}$ does not prevent transcriptional read through when in the same orientation as the target gene. When cloning into the pTOPO plasmid, $\mathrm{kan}^{\mathrm{R}}$ present in the cloning vector was inactivated by digestion with NcoI and end-filling of the DNA ends with Klenow enzyme and dNTPs. Following re-ligation the plasmid was transformed into E. coli DH5 $\alpha$. Genes HIO144 $(n a n K)$ and HIO145 (nanE) were amplified together using the primers 0145 for and 0143rev (Table 1) and each gene was then inactivated independently by

\section{catabolism transport}

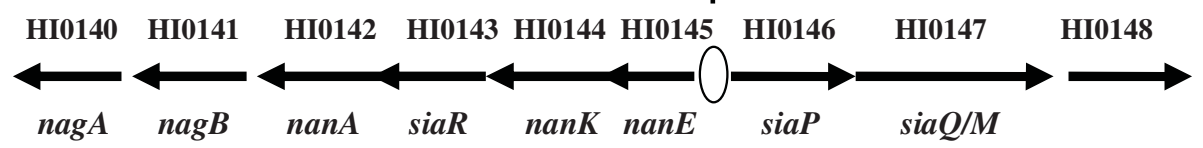

Figure 1 The sialometabolism gene cluster of $\boldsymbol{H}$. influenzae. Indicated are the catabolism and transport groups of genes, each gene is represented by an arrow indicating the direction of transcription. The HI numbers corresponding to the reading frame designation in the strain Rd genome sequence are given above the arrows and the gene names below. 0 indicates the position of the CRP binding sequence. 
Table 1 Oligonucleotide primers used in this study.

\begin{tabular}{|c|c|c|c|}
\hline primer & Sequence $\left(5^{\prime}-3^{\prime}\right)$ & primer & Sequence $\left(5^{\prime}-3^{\prime}\right)$ \\
\hline 0140for & CTGCAATTAAATGGCTGTGG & 0140rev & GCAATTGTGTCATTCGCATC \\
\hline 0141 for & TCAGTTGTTGGGCTGCAC & 0141rev & CAGCAACTGCGCCTTCTA \\
\hline nanAfor & TCCGCCATAATATCGACAAA & nanArev & TTTGCTTTTGCAAGCTGTTC \\
\hline 0143 for & AATTGCCGATACGATTITGC & 0143rev & TATCTTCTTCGCCCTGCACT \\
\hline 0144for & TGCGTTGTTTAGCACTAG & 0144rev & GCTAATCCCACACTGCCA \\
\hline 0145 for & TTGCCAACCTGTCGATGA & 0145rev & CCCTCAGCCATCACAAAACA \\
\hline 0146for & TGTTCTTGCCGCTGATTATG & 0146rev & CATTITCGGCAGCATCTITT \\
\hline 0147for & GGAGTGAAGAACTCGCCAAC & 0147rev & TCACGCATTGCTITGATTT \\
\hline 0148for & TITITCAGCGAACGCACA & 0148rev & TCAGTITCACCGCCAATCA \\
\hline FRDL & СCCTCAATTTGGTTAAATCCTG & FRDR & CCATGGTCACGGTTATCAAGA \\
\hline HI1045L & CAAGAAGTGCTTTCTCAAATTCAA & $\mathrm{HI0145R}$ & TTTATCCATTGGGCCATCAT \\
\hline HI0146L & TCTGACTITACCTITGCAGAAT & $\mathrm{HI0146R}$ & AATACTGCCGCTTCAGGGTA \\
\hline HI0143L & AAATCGCAAAACAAAATGGTG & HI0143R & CGGGGGAACGCAAACTAT \\
\hline crpA & GCAACTCAACGAGATCCC & crpD & GACCAATCCTGTCTTCCT \\
\hline$\overline{\text { nagE }}$ & GAACCGCCCACATATAAG & nagF & TGCGTTGTTIAGCACTAG \\
\hline
\end{tabular}

insertion of $\mathrm{kan}^{\mathrm{R}}$ at NruI and BglII sites respectively. For nanA (HI0142), insertion of $\mathrm{kan}^{\mathrm{R}}$ was achieved following partial digestion with Mfe1 and siaR (HIO143) was inactivated by inserting $\mathrm{kan}^{\mathrm{R}}$ at an MfeI site.

Mutant $H$. influenzae strains were constructed following transformation [21] of strain RM118, NTHi 375 or 486 using the appropriate plasmids that had been linearized by restriction endonuclease digestion. The resulting mutant strains were confirmed as correct after growth on $\mathrm{BHI} /$ kanamycin and by both $\mathrm{PCR}$ and restriction digestion analyses.

\section{Analysis of LPS by electrophoresis}

Bacterial lysates were prepared from cells grown overnight on BHI plates to which Neu5Ac had been added. Lysates were then analyzed by tricine-SDS-PAGE and staining with silver as described previously [22].

\section{Serum bactericidal assay}

Bacteria cultured on BHI plates to which Neu5Ac has been added were assayed for killing by pooled human serum, as described previously [2].

\section{RT-PCR analysis}

Bacteria were cultured in BHI or CDM medium, with or without added Neu5Ac. When the $\mathrm{OD}_{600}$ reached 0.3 (CDM) or 0.6 (BHI), $1 \mathrm{ml}$ aliquots of cells were collected and added directly to $2 \mathrm{ml}$ RNA Protect Bacterial Reagent (Qiagen) and RNA was extracted using a SV Total RNA Isolation Kit (Promega). cDNA was synthesized by adding approximately 500 ng RNA, quantified by a Nanodrop spectrophotometer, to $2 \mu \mathrm{l}$ Random Primers (Promega, $500 \mu \mathrm{g} \mathrm{ml}^{-1}$ ), $1 \mu \mathrm{l}$ RNasin (Promega, 40
$\mathrm{U} \mu \mathrm{l}^{-1}$ ), and the volume made up to $12 \mu \mathrm{l}$ with DEPCtreated water. After heating at $70^{\circ} \mathrm{C}$ for $10 \mathrm{mins}$ the sample was cooled on ice and a $1 \mu \mathrm{l}$ aliquot removed to be used in a control PCR to ensure that the sample was DNA free. A mix of $4 \mu \mathrm{l}$ DEPC water, $5 \mu$ l of $5 \times$ Buffer (Invitrogen), $1 \mu \mathrm{l}$ dNTP's (25 mM Invitrogen), $2 \mu \mathrm{l}$ of $0.1 \mathrm{M}$ DTT (Invitrogen) and $1 \mu \mathrm{l} \mathrm{M}$-MLV-Reverse Transcriptase (Invitrogen, $200 \mathrm{U} \mathrm{\mu l}^{-1}$ ) was added to the reaction and incubated at $37^{\circ} \mathrm{C}$ for 1 hour followed by $95^{\circ} \mathrm{C}$ for 5 mins. $1 \mu \mathrm{l}$ of cDNA was then used as template in subsequent PCR reactions (RT-PCR), carried out using the conditions described above, or in realtime quantitative PCR (q-PCR). q-PCR reactions were performed in triplicate using the Corbett Research Rotor Gene RG-3000. Each reaction was performed in an individual tube and made up to $25 \mu$ l containing 5 $\mu \mathrm{l}$ cDNA, $12.5 \mu \mathrm{l}$ PCR Master Mix (Abgene), $0.25 \mu \mathrm{l}$ probe, $1 \mu \mathrm{l}$ of forward and reverse primer and $5.25 \mu \mathrm{l}$ $\mathrm{H}_{2} \mathrm{O}$. Conditions for the q-PCR reaction were $2 \mathrm{~min}$ at $50^{\circ} \mathrm{C}, 10 \mathrm{~min}$ at $95^{\circ} \mathrm{C}$ and then 40 cycles, each consisting of $15 \mathrm{~s}$ at $95^{\circ} \mathrm{C}$, and $1 \mathrm{~min}$ at $60^{\circ} \mathrm{C}$. The housekeeping gene, $f r d B$, was used as the reference gene. Left (L) and Right (R) primer pairs for genes frdB, siaR, nanE and siaP are given in Table 1 . Probe \#s 3, 59, 137 and 59 (Roche) were used respectively in the q-PCR reactions for these genes. Relative quantitation of gene expression was performed using the method described by Pfaffl [23]. Results given are based on the mean value of PCRs performed in triplicate in the same experiment. q-PCR was repeated a minimum of three times for each gene using independent cDNA and mRNA preparations from different batch growths of bacteria. 


\section{Chinchilla model of Otitis Media}

An experimental chinchilla (Chinchilla lanigera) model of acute OM was used [24]. Animal care and all related procedures were performed in accordance with institutional and federal guidelines and were conducted under an Institution Animal Care and Use Committeeapproved protocol at Boston University Medical Centre [3]. Wild type NTHi 375, 486 and RM118 and their respective isogenic mutant strains (nanA, siaR, siaP, crp) were grown overnight for 16 hours in BHI broth. For animal challenge, the overnight grown bacteria were diluted in Hank's balanced salt solution (HBSS) and approximately 50-100 c.f.u. in $100 \mu \mathrm{l}$ were inoculated through the left superior bulla of adult chinchillas with a 25-gauge tuberculin needle $[3,5]$. After seventy-two hours, tympanometry, otomicroscopy, and middle ear cultures were performed to determine if infection was present. The middle ear cavity was accessed and a direct culture was obtained as described previously [5,24]. Middle ear fluid (MEF) when present was obtained and if MEF was absent the middle ear was flushed with HBSS, 10-fold serial dilutions were prepared as previously described [3,5]. A volume of $100 \mu$ of each dilution was plated onto chocolate agar plates (Remel). The lower limit of detection of viable organisms in MEF using this dilution series is 100 c.f.u. $\mathrm{ml}^{-1}$ [3]. Direct and indirect examination of the ears was performed on days 3, 7, 12, and 19 following inoculation with NTHi strains, and days 3, 7 and 11 following inoculation with strain $\mathrm{Rd}$, or until the middle ear cultures were sterile on two consecutive samples. The median bacterial density was calculated for each organism at each sample point and statistically significant differences were determined using the Wilcoxon Rank-Sum Test (SaS 9).

\section{Results}

\section{The genes of the sialometabolism region are conserved} in $H$. influenzae

Previous studies by us using a $H$. influenzae whole genome microarray [25] and by others [12] identified a region of DNA comprising nine contiguous genes that encode functions relating to sialometabolism (Figure 1). The genes for sialic acid catabolism (HIO140 (nagA), HIO141 (nagB), HIO142 (nanA), HIO144 (nanK), HIO145 (nanE) and including HIO143 (siaR)) and procurement (HI0146 (siaP), HI1047 (siaQM), HIO148) are transcribed divergently (Figure 1). siaR and nanK possess overlapping ORFs whilst three pairs of adjacent genes have intergenic regions of $<50 \mathrm{bp}$.

To explore how general this arrangement of the sialometabolism region of DNA is in $H$. influenzae, we examined $25 \mathrm{NTHi}$ isolates selected because they are epidemiologically distinct and representative of NTHi genetic diversity [17]. All 25 isolates incorporate sialic acid into their LPS as a terminal residue [26], although the location and amount of Neu5Ac in LPS glycoforms, and the repertoire of sialyltransferase genes present, are variable between strains. PCR analysis was carried out on chromosomal DNA from each strain using internal primers for each of the 9 genes (HIO140-HIO148) (Table 1) and primers designed against genes in the flanking regions (HIO139 encoding P2 protein on the 5' side and HIO148.1/HIO149 on the 3' side). This analysis confirmed that both the presence of individual sialometabolism genes and their organization in all $25 \mathrm{NTHi}$ strains was conserved and overall was the same as that of strain Rd (Figure 1). H. influenzae type b strains also maintained the sialometabolism gene cluster (data not shown). Two of the twenty five NTHi strains, 375 and 486, which have been used in previous in vitro and in vivo studies of sialic acid metabolism, were selected for further investigation together with strain Rd. Mutations in genes within the sialometabolism region of DNA in strains Rd, 375 and 486, with the exception of nagA and $n a g B$, were made. $n a g B$ encodes the last of the five steps of the Neu5Ac catabolic pathway (converting glucosamine-6-phosphate to fructose-6-phosphate), suggesting that the gene product may be essential because of its close association with central metabolism, as had been previously described for nagA [27].

\section{The sialometabolism uptake genes are essential for LPS sialylation and virulence}

$H$. influenzae possesses two genes, siaP (HI0146) and siaQ/M (HIO147) separated by $60 \mathrm{bp}$, which encode a two component TRAP transport system $[10,11]$. The phenotypic effect of mutation of siaP and siaQ/M on LPS structure of NTHi strains was analyzed using gel electrophoresis. In agreement with previous studies using strain Rd [10] and NTHi 2019 [12], siaP and siaQ/M mutants of NTHi strains 375 and 486 showed altered mobility of LPS consistent with a loss of sialylated LPS glycoforms when compared to the respective wild type (Figure 2). Further, the siaP mutant of strain 486 showed no change in LPS profile upon neuraminidase treatment (Figure 2). These data are fully consistent with the TRAP transporter being the primary means of sialic acid uptake in these NTHi strains.

Sialylation of LPS [28] is known to be an important virulence factor in $H$. influenzae, conferring increased resistance to killing by normal human serum $[2,3]$. There was a marked decrease in the survival of mutants deficient in sialic acid uptake compared to wild type for strains Rd (Figure 3a), 486 (Figure 3b) and 375 (data not shown) following exposure to pooled human serum for 45 mins, in agreement with previously published data [10].

By comparison, for strain $\mathrm{Rd}$, the phenotype of a RdnanE mutant, affected in Neu5Ac catabolism, was 


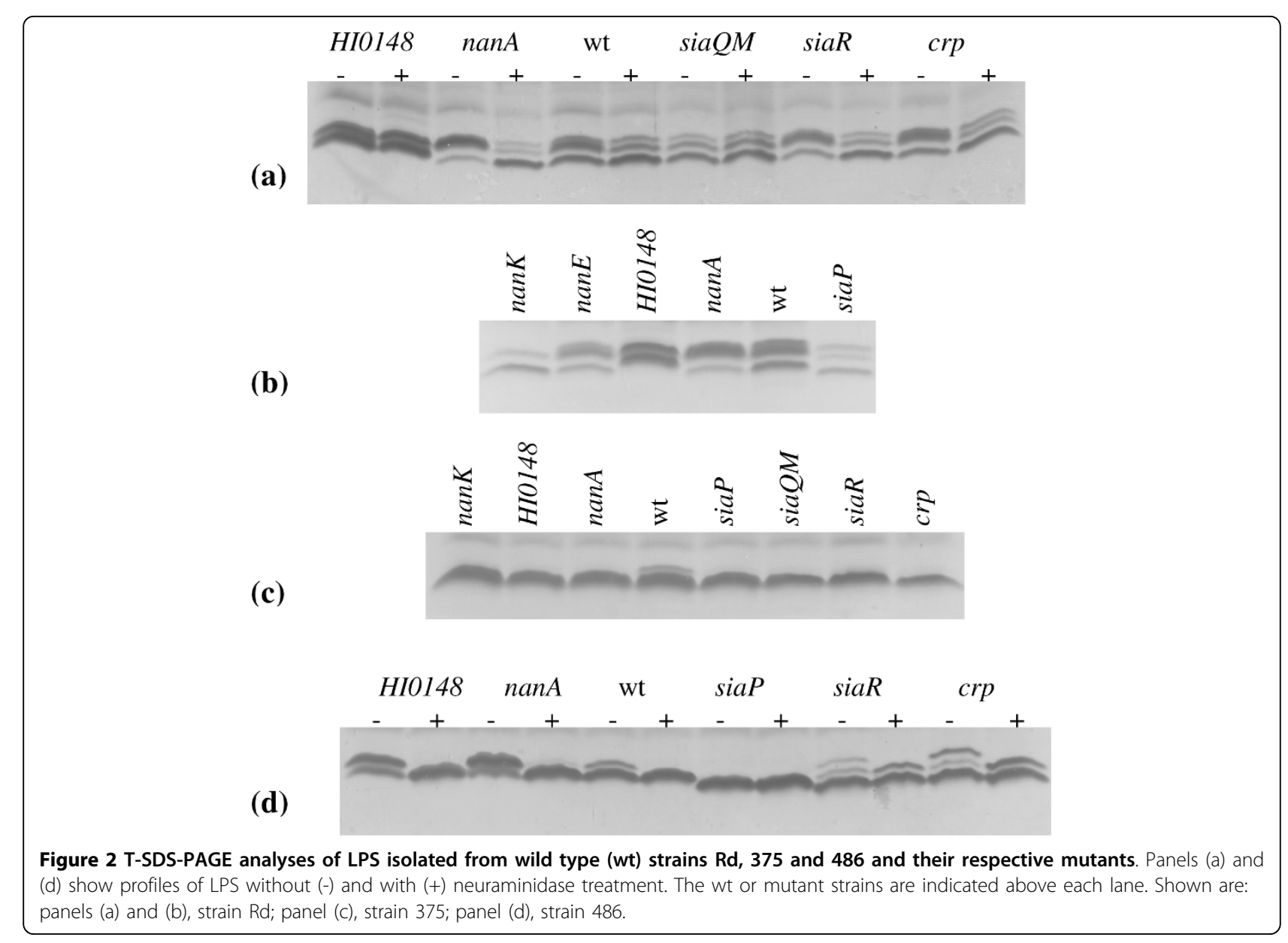

relatively unchanged compared to wild type based on electrophoresis of LPS (Figure 2b) and susceptibility to killing in a bactericidal assay (Figure 3b). However, when a $\operatorname{Rd}$ nanA mutant was compared to wild type by SDS-PAGE it was hypersialylated (Figure 2a) and showed increased serum resistance to killing when compared to the parent strain (Figure 3a). The changes in LPS profile when comparing the wild-type to strains with mutations in sialic acid catabolism genes in the 486 and 375 backgrounds were generally similar to the changes observed for strain Rd (data not shown).

NTHi strains 375 and 486 have previously been used to investigate the role of sialic acid as a virulence factor in a well described chinchilla model of OM [3,5]. For NTHi strains 375, 486 and strain Rd, we compared wild type and siaP mutants; approximately 100 c.f.u. were inoculated directly into the bullae of four animals in each group, with the exception of 375 wild type (2 animals). For strains $\mathrm{Rd}$ and 486, siaP mutants with a deficient TRAP transport system were clearly attenuated, with low or undetectable bacterial counts in the middle ear after two days (Figure 4). All middle ears (100\%) inoculated with strains 486 and Rd developed high- density infection compared to the absence of middle ear disease in animals challenged with siaP mutants; 486 siaP ( $0 / 4$ ears culture positive; $\mathrm{p}=0.02)$, RdsiaP $(0 / 4$ ears culture positive; $\mathrm{p}=0.03$ ). For strain 375 , the attenuation was less marked (Figure 4) and not statistically significant for the siaP mutant compared to the wild-type strain (375siaP $3 / 6$ ears culture positive; $\mathrm{p}=$ 0.39 , but sample for 375 wild type was from only 2 animals). This is possibly due to the low levels of LPS sialylation observed for strain 375. Strain RdnanA which showed enhanced LPS sialylation in vitro was of equivalent virulence to the parent strain in the middle ear of the chinchilla (Figure 4) (no statistically significant difference between $\mathrm{Rd}$ and $\mathrm{Rd} n a n A$ (4/4 ears culture positive; $\mathrm{p}=0.31)$ ).

\section{Sialylation of $H$. influenzae LPS is adaptive and is subject to complex regulation}

Sialic acid may be incorporated into LPS or utilized as a source of carbon and nitrogen for NTHi. In the host, given the context of the complex array of other potential nutrients available to $H$. influenzae and the two potential fates for Neu5Ac in the bacterium, it is 

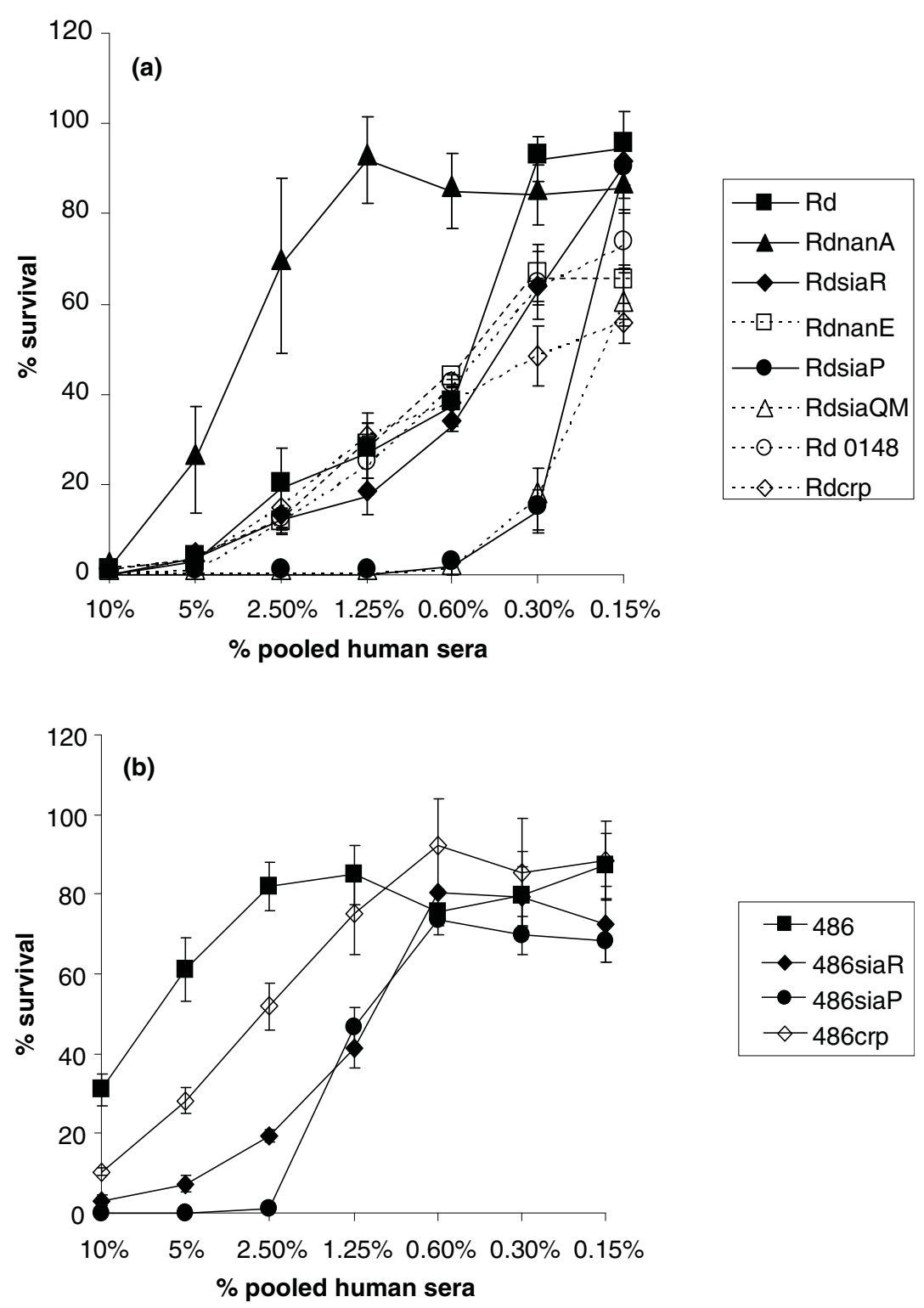

Figure 3 Resistance (\% survival) of $\boldsymbol{H}$. influenzae strains to the killing effect of normal human serum. 500 organisms of strain Rd (panel a) or NTHi 486 (panel b) or derived mutants were added to different (doubling) dilutions of pooled human serum; percentage survival of inoculum of bacteria (y-axis) is shown for varying serum concentrations ( $x$-axis). Each point is the averaged result of 3 independently performed experiments, error bars (1 standard deviation) are shown.

reasonable to assume that sugar utilization in $H$. influen$z a e$ is regulated at the genetic level. The intervening 353 bp between the sets of divergently transcribed sialometabolism genes include the binding sites for the regulatory proteins SiaR and CRP [12].

In our experiments, mutation of siaR showed somewhat different phenotypes dependent upon the strain background. Compared to wild type, the RdsiaR mutant strain showed little difference in LPS phenotype (Figure $2 \mathrm{~d}$ ), but was slightly more susceptible to killing in the serum bactericidal assay following growth in the presence of added exogenous sialic acid (Figure 3a). A reduction of serum resistance of a 486 siaR mutant (Figure $3 \mathrm{~b}$ ) compared to the parent strain is consistent with some LPS truncation (Figure 2d), although the reason for this is unknown. No significant difference between strains 375 and 375 siaR was seen for LPS phenotype (Figure 2c) or in the bactericidal assay (data not shown). Importantly, in the chinchilla model of OM, mutation of siaR in strains $\mathrm{Rd}, 375$ and 486 produced strains that were virulent (Figure 4), although we cannot rule out some difference in bacterial titres during the course of 

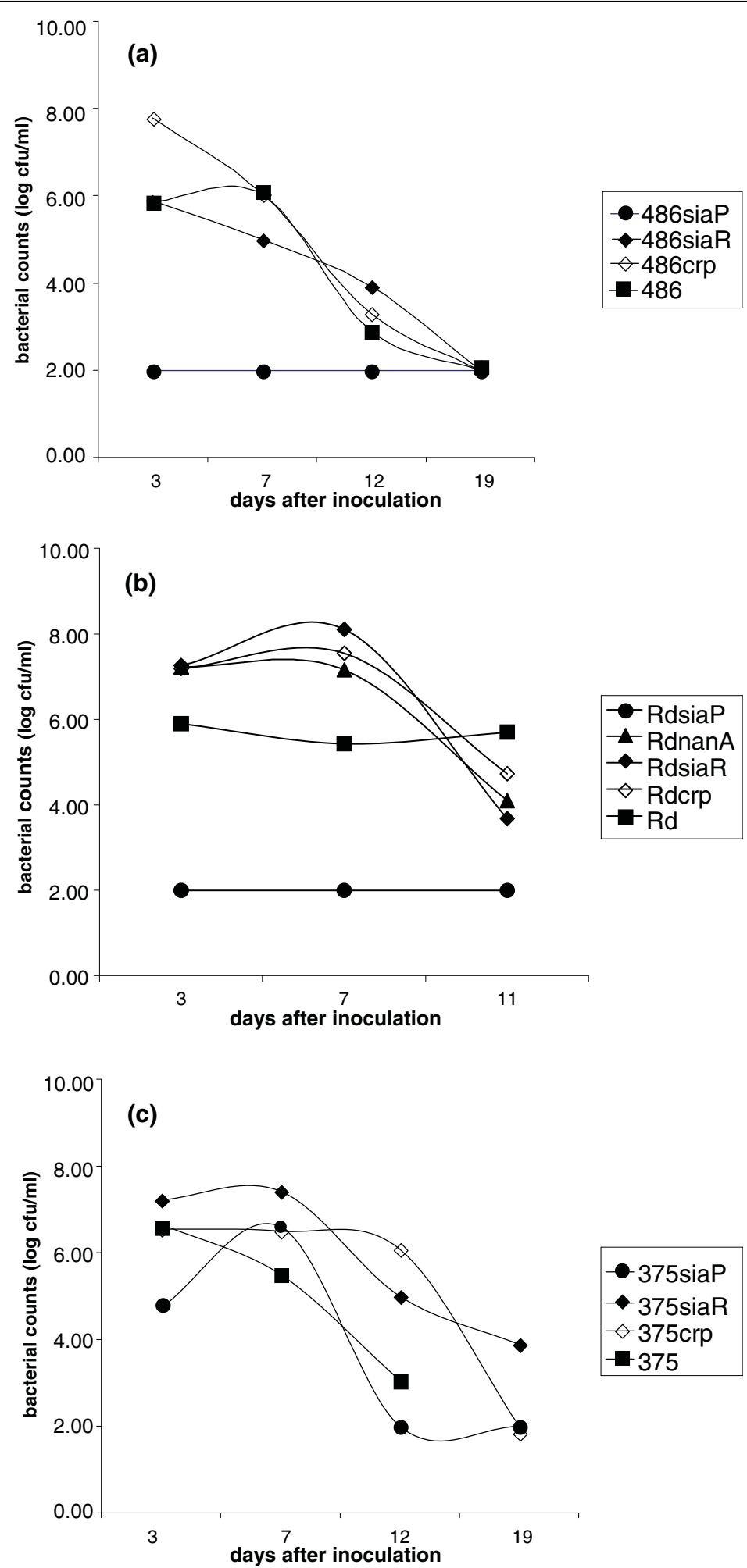

- 375 siaP

-375 siaR

$\diamond 375 \mathrm{crp}$

375

Figure 4 Effect of mutation of siaP, siaR and crp on bacterial counts of $H$. influenzae strains from the middle ear of chinchillas when compared to wild type strains. Animals were inoculated with between 60 and 100 organisms directly into the middle ear bullae. Each data point represents the average number of organisms $\mathrm{ml}^{-1}$ of exudate or washings from the middle ear for typically four animals at different times (days) following inoculation. Shown are wild type and isogenic strains for: panel (a), NTHi 486; panel (b), Rd; panel (c), NTHi 375. The lower detection limit is a bacterial count of 2.00 
disease. Thus, siaR is not essential for virulence in this model.

There is a consensus sequence for CRP binding (TGTGATCAACTTCTCA) within the DNA region intergenic between $n a n E$ and siaP [12,29], consistent with the role of CRP in regulating Neu5Ac uptake genes. Of the mutant strains with crp inactivated, only NTHi 486 displayed any alteration in LPS profile (Figure $2 \mathrm{~d}$ ) and some increased serum sensitivity compared to the parent strain (Figure 3b). Significantly, in vivo in the chinchilla, each of the strains Rdcrp, 375 crp and 486 crp were virulent (Figure 4).

To investigate in more detail the interdependence of genes involved in sialometabolism, we compared gene expression in wild type and mutant strains following growth in the presence or absence of exogenous Neu5Ac. RT-PCR analysis of total RNA extracted from strain $\mathrm{Rd}$ mutated in each of the genes nanA, siaR, nanK, nanE, siaP, siaQM, HIO148 and crp was performed using internal pairs of primers specific for each gene of interest (Table 1) and the levels of expression compared using the RT-PCR amplification product for the housekeeping gene, $f r d B$, as a control between samples. The level of transcript for each sialometabolism gene was generally greater in the siaR mutant background when compared to the wild type strain, although the results proved difficult to quantify (data not shown). This would be consistent with SiaR exerting a regulatory (negative) effect on sialometabolism gene expression, i.e. acting as a repressor [12]. The corresponding change in expression of multiple genes might suggest some co-regulation or co-dependence. Using primer pairs targeted against the 5' and 3' ends of adjacent genes across the region, RT-PCR analysis showed some co-transcripts for most gene pairs across the sialometabolism region (Figure 5).
We obtained quantitative data for the changes in the level of expression of representative sialometabolism genes (siaR, nanE, siaP, HI0148) by q-PCR. These data confirmed the key observation from our initial microarray experiment [25], i.e. that there is a small but consistent two to three fold repression of sialometabolism gene expression following growth of strain $\mathrm{Rd}$ in the presence of exogenous sialic acid in both BHI and the chemically defined (CDM) media (Figure 6, Table 2). Quantification of the changes in transcript levels of the first gene of each of the divergently transcribed sialometabolism regions $n a n E$ (catabolic) and siaP (transport) in the siaR mutant background showed 11 and 13 fold increased expression levels respectively when compared to the parent strain following growth in the absence of added Neu5Ac (Figure 6) confirming that SiaR acts to repress both the catabolic and uptake genes. Changes in gene expression in response to exogenous Neu5Ac, however, were not evident in the siaR mutant strain (Figure 6) although siaR expression was itself slightly repressed (2 fold) following growth of the wild type strain in the presence of sialic acid. A transcript for the siaR gene was unexpectedly detected from the siaR mutant strain in both our q-PCR and RT-PCR experiments; in the latter, the size corresponded to that of the native gene. DNA sequencing of this cDNA revealed that $\mathrm{kan}^{\mathrm{R}}$ had been deleted leaving a $1 \mathrm{bp}$ insertion that constituted a frameshift of the siaR ORF. The reason for the apparent instability of $\mathrm{kan}^{\mathrm{R}}$ in this gene following reverse transcription is not understood. The siaP gene showed a significant 8 fold increase in expression in the nanE mutant strain compared to the parent strain, following growth without added Neu5Ac (Figure 6).

The most significant change in gene expression detected in a crp mutant in the Rd strain background was for the siaP gene, expression was decreased 19 fold when

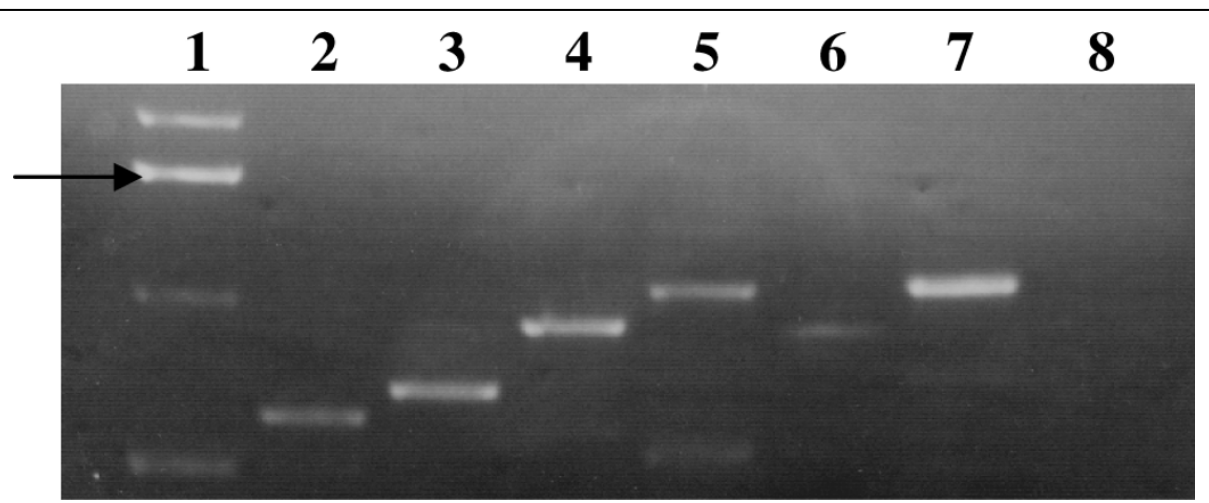

Figure 5 PCR amplification for CDNA of sialometabolism genes from strain Rd showing co-transcripts for adjacent gene pairs. CDNA was made after bacteria were grown in BHI in the presence of sialic acid. RT-PCR products shown are in lane 2, nagA/nagB; lane 3, nagB/nanA; lane 4, nanA/siaR; lane 5, siaR/nanK; lane 6, nanK/nanE; lane 7, siaP/siaQM; lane 8, siaQM/HI0148. Lane 1 shows the 1 kb DNA ladder marker with the $1.6 \mathrm{~kb}$ band marked by an arrow. 


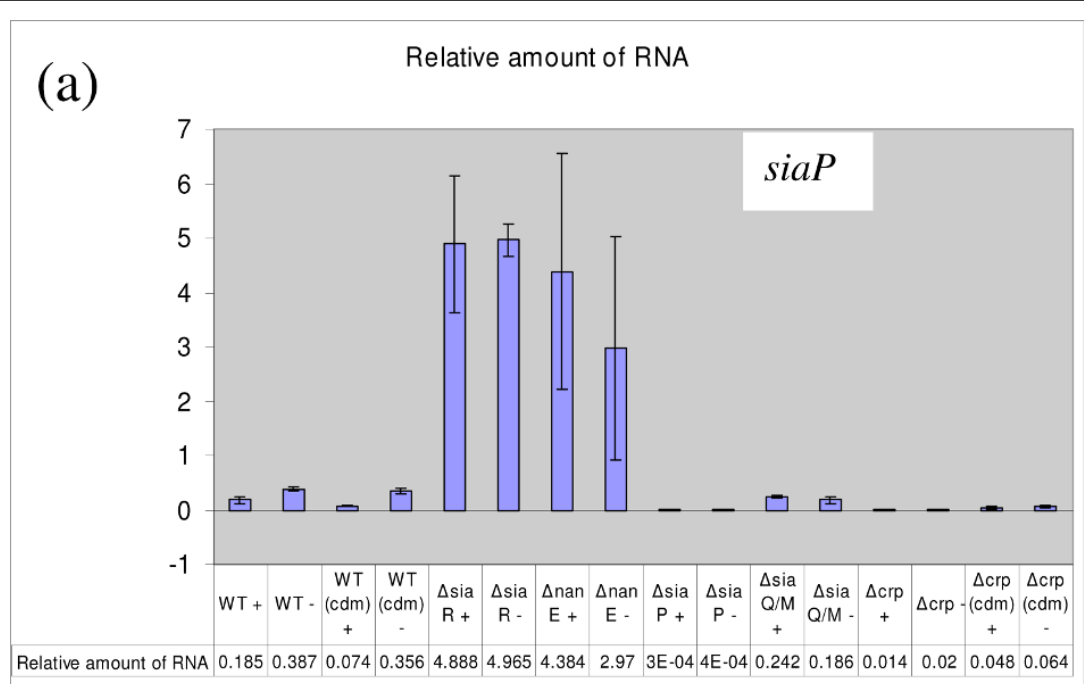

(b)

Relative amount of RNA

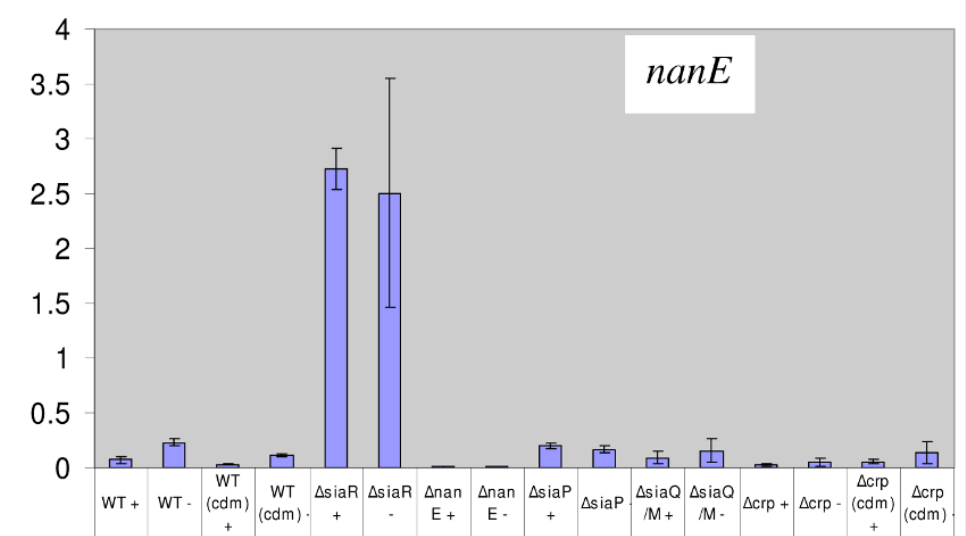

\begin{tabular}{ll|l|l|l|l|l|l|l|l|l|l|l|l|l|l|l} 
Relative amount of RNA & 0.072 & 0.23 & 0.029 & 0.111 & 2.724 & 2.502 & 0.012 & 0.011 & 0.195 & 0.166 & 0.092 & 0.153 & 0.025 & 0.054 & 0.052 & 0.136
\end{tabular}

(c)

Relative amount of RNA

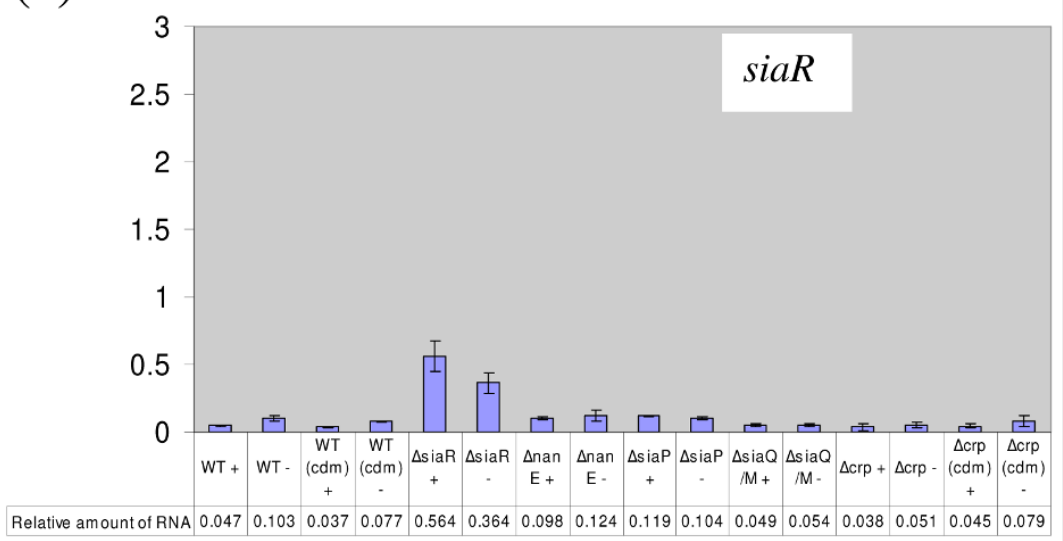

Figure 6 q-PCR data for sialometabolism genes of $\boldsymbol{H}$. influenzae. In each panel, the $y$-axis shows the quantity of mRNA, relative to the frdB control gene, for CDNA from wild type or mutant strains following growth in the presence (+) or absence (-) of exogenous Neu5Ac ( $x$-axis). Shown are: panel (a) siaP; panel (b) nanE; panel (c) siaR. Each value shown below the $\mathrm{x}$ axis represents the results from 3 separate experiments utilising independent CDNA and mRNA preparations and each q-PCR reaction was run in triplicate. The error bars indicate the standard deviations derived for the respective data. 
Table 2 Transcription analyses of sialometabolism genes in $\mathbf{R d}$ and derived mutant strains.

\begin{tabular}{llll}
\hline \multirow{2}{*}{ strain } & \multicolumn{3}{c}{ Gene expression ratio: } \\
\cline { 2 - 4 } Rd & siaP & nanE & siaR \\
\hline siaR & 2.1 & 3.2 & 2.2 \\
\hline nanE & 1.0 & 0.9 & - \\
\hline siaP & 0.7 & - & 1.3 \\
\hline siaQ/M & - & 0.9 & 0.9 \\
\hline crp & 0.8 & 1.7 & 1.1 \\
\hline Rd (CDM) & 1.4 & 2.2 & 1.3 \\
\hline Values given are & 4.8 & 3.8 & 2.1 \\
\hline
\end{tabular}

Values given are for the ratio of the expression level of the gene following growth in $\mathrm{BHI}$ in the absence of added Neu5Ac to growth with added Neu5Ac, taken from the data given in Figure 6. Also shown are the values for strain Rd following growth on CDM medium. A dashed line indicates no expression following inactivation of the respective gene.

compared to the parent strain following growth in the absence of Neu5Ac (Figure 6). A similar reduction was observed following growth on both BHI and CDM media, although the magnitude of the change was less on CDM. No response to the presence or absence of Neu5Ac in the medium was observed for siaP expression in strain Rdcrp. No significant change in either nanE or siaR expression was found in strain Rdcrp with respect to the parent strain following growth on CDM, although an up to 4 fold change was observed using BHI medium (Figure 6). These data are consistent with the previous observation that CRP has a differential effect on sialometabolism genes, having a preferential role in activating uptake rather than catabolic genes [12].

$H I 0148$, the gene downstream of siaQ/M encodes a protein that contains six Kelch motifs that are often associated with sialic acid binding proteins such as neuraminidase enzymes [30]. A RdHIO148 mutant strain showed some loss of the lowest molecular weight glycoforms (Figure 2), but no difference in serum sensitivity (Figure 3), when compared to the wild type. No significant change in sialometabolism gene expression was observed following mutation of HIO148 (data not shown).

\section{Discussion}

Sialic acids are a diverse family of sugars and are components of bacterial surface macromolecules such as capsular polysaccharides and glycolipids that are of major biological importance in pathogenesis. In $H$. influenzae, Neu5Ac is a potential carbon and energy source $[8,12]$ as well as a component of the LPS of almost all NTHi strains where detailed structure has been determined to date [26,31-33]. H. influenzae lacks the genes required for the synthesis of Neu5Ac and in nature must acquire it from humans, its only natural host. It has been shown that $H$. influenzae acquires Neu5Ac during experimental infection of chinchillas and that its incorporation into LPS is critical for virulence [3]. It has been estimated that the concentration of Neu5Ac potentially available in human tissues and fluids is $0.5 \mathrm{mg} / \mathrm{ml}$ [8] making it a potential major nutrient for the bacterium in vivo.

In the present study we have investigated genes involved in the dynamic interplay between utilisation of Neu5Ac in the biosynthesis of LPS (sialylation) or its potential as a catabolite. Microarray [25] and bioinformatic $[8,12]$ analyses had identified a set of 9 contiguous genes that played a significant role in sialometabolism. We reasoned that an investigation of the transcription of $H$. influenzae sialometabolism genes would provide further insights into the genetic regulation relating to sialometabolism. Our study presents a number of novel or different findings from the study of Johnston and colleagues [12], including the effect of Neu5Ac in modulating transcription of sialometabolism genes, the conserved organisation of the sialometabolism genes, and the effects of mutation of the regulatory genes, siaR and crp, on experimental infection in a chinchilla animal model of OM.

The sialometabolism locus consists of nine genes, organized such that divergently transcribed catabolism and transport genes, are separated by an intergenic, non-coding region of $353 \mathrm{bp}$. This intergenic region contains a consensus CRP binding site and an overlapping site to which SiaR binds [12]. In the NTHi strain (2019) studied by Johnston and colleagues, they conclude that exogenous Neu5Ac did not affect transcription of either the catabolic or transporter sialometabolism genes. In contrast, we have observed Neu5Ac-dependent transcriptional down-regulation when $H$. influenzae was grown in both $\mathrm{BHI}$, a relatively complex medium, and CDM, a more defined medium. The transcriptional down-regulation of both transporter and catabolic genes that we had previously observed using DNA microarrays has now been confirmed and quantified by q-PCR. As an important indication of the general significance of sialometabolism to $H$. influenzae biology, the present study provides molecular epidemiological evidence that the sialometabolism gene cluster is conserved across a set of NTHi strains that are representative of the genetic diversity found in the natural population of NTHi [17]. This genetic conservation of sialometabolism genes between strains is in contrast to the well documented inter-strain LPS structural diversity that includes the variable location and stoichiometry of Neu5Ac, which is characteristic of NTHi strains [26,33].

Sialometabolism genes are found clustered in many other bacterial species [9]. siaR homologues exist in other proteobacteria, e.g Pasteurella $s p$. but in the context of a different gene organisation [9]. In Pasteurella 
multocida, a pathogen of cattle and birds, the sialic acid TRAP transporter genes are located adjacent to catabolic genes that have a somewhat different gene organisation to $H$. influenzae [34].

Details of the mechanism(s) by which exogenous Neu5Ac alters transcriptional activity in $H$. influenzae remains unclear. Purified SiaR protein has been investigated by Johnston and colleagues [12] and has been demonstrated to bind to the intergenic region to downregulate transcription of genes for the uptake and catabolism of sialic acid. Using RT-PCR and q-PCR in different strains of $H$. influenzae, we provide corroborating evidence that there is increased transcription of sialometabolism genes when siaR is disrupted. Mutation of siaR in our study resulted in up to a 19 fold increase in expression of sialometabolism genes tested. These changes are of a similar magnitude to the increased expression of the sialometabolism genes (range 2 to 16 fold) compared to the parent strain observed by Johnston and colleagues in a siaR mutant of NTHi 2019 [12]. A reasonable hypothesis is that the SIS domain [14] present in the SiaR protein could be a binding site for Neu5Ac, or perhaps other related sugars (e.g. Nacetylglucosamine or glucosamine-6-phosphate), that activate(s) the repressor activity of SiaR. Our findings from q-PCR provide clear evidence of a role for CRP as a positive transcriptional activator through its interaction with the consensus binding site located in the intergenic region in the middle of the sialometabolism genes, findings in agreement with previous studies [12]. In contrast to SiaR, which affects both catabolic and transporter genes, CRP is a positive transcriptional regulator primarily of Neu5Ac uptake.

An unexplained and intriguing aspect of sialometabolism in H. influenzae is the potential role for the HI0148 protein. The HI0148 protein contains Kelch motifs and recent studies in E. coli have shown that a homologue of the HI0148 protein, NanM, functions as a Neu5Ac mutarotase [35]. This mutarotase converts $\alpha-\mathrm{Neu} 5 \mathrm{Ac}$ to the $\beta$ - form and vice versa. In solution, free Neu5Ac will tend to spontaneously shift towards the $\beta$-form. It is an interesting possibility that HI1048 could provide the correct anomer of Neu5Ac for uptake, or perhaps for catabolism or regulation. The function of NanM in $H$. influenzae is currently under investigation.

The crucial role of sialylation of LPS in the pathogenesis of $H$. influenzae infection has been demonstrated in a chinchilla model of OM [3]. Sialylation of NTHi LPS interferes with the binding, activation and immune clearance of $H$. influenzae effected by complement components [5]. Mutant strains in which the Neu5Ac TRAP uptake system has been disrupted (e.g. siaP mutants) are deficient in LPS sialylation and we show here that these mutants are attenuated, although the degree of attenuation was greater for strains 486 and $\mathrm{Rd}$ than for 375. This finding emphasises the complexity of the mechanisms affecting host immune clearance but are broadly consistent with the relatively decreased LPS sialylation of strain 375 when compared to strain 486 [2]. Disruption of the TRAP transport system in P. multocida similarly attenuated bacterial virulence in the mouse [34] and turkey [36] models of systemic infection.

In contrast to the attenuation of siaP mutants in each of three $H$. influenzae strains tested, mutation of the genes encoding both the regulatory proteins SiaR and Crp showed no or little effect on virulence over the course of a 19 day infection in the chinchilla. We have shown that LPS remains sialylated in each of these mutant strains. Analysis of the sialylation profiles of the LPS isolated directly from bacteria taken from the middle ears of animals infected with these mutant strains could provide critical supportive in vivo evidence of LPS sialylation. Future studies should use an ascending model of infection in which infection is initiated through inoculation of the nasopharynx. The more relevant selection pressures contributing to the evolution of LPS sialylation and its regulation are likely to be a function of $H$. influenzae fitness for carriage and transmission rather than its role in disease. An understanding of the role of sialic acid, provided by the host, to the commensal and virulence lifestyles of $H$. influenzae would provide valuable insights into an aspect of host microbial interaction that might provide novel targets for intervention in disease caused by this bacterium.

\section{Conclusion}

Expression of a set of genes required for sialometabolism in $H$. influenzae is altered through growth of the bacteria in the presence of sialic acid. Mutation of representative genes influences sialylation of the LPS molecule. The regulation of sialometabolism gene expression is complex but there appears to be no major requirement for the positive (CRP-dependent) or negative (SiaR-dependent) transcriptional regulation on LPS sialylation in experimental OM induced through direct inoculation of organisms into the middle ear of chinchillas.

\section{Acknowledgements}

GAJ and DWH were supported by grants from the Medical Research Council, UK and GAK from the Wellcome Trust. We thank Michael Apicella and Jason Johnston for helpful comments on the manuscript.

\section{Author details}

'Molecular Infectious Diseases Group, University of Oxford Department of Paediatrics, Weatherall Institute of Molecular Medicine, John Radcliffe Hospital, Headington, Oxford, OX3 9DS, UK. ${ }^{2}$ Maxwell Finland Laboratory for Infectious Diseases, Division of Pediatric Infectious Diseases, Boston University School of Medicine, Boston Medical Center, 774 Albany Street, Boston, MA 02118, USA. 


\section{Authors' contributions}

GAJ helped to design and carried out the transcription experiments, WAS analysed the combined data and helped to draft the manuscript, KM carried out the LPS gel and SBA analyses, GAK carried out the q-PCR analysis, MAF and SIP designed and carried out the chinchilla experiments and helped draft the manuscript, ERM and DWH conceived the study and helped analyse the data and draft the manuscript. All authors read and approved the final draft.

Received: 25 June 2009

Accepted: 16 February 2010 Published: 16 February 2010

\section{References}

1. Varki A: Biological roles of oligosaccharides: all of the theories are correct. Glycobiology 1993, 3(2):97-130.

2. Hood DW, Makepeace K, Deadman ME, Rest RF, Thibault P, Martin A, Richards JC, Moxon ER: Sialic acid in the lipopolysaccharide of Haemophilus influenzae: strain distribution, influence on serum resistance and structural characterization. Mol Microbiol 1999. 33(4):679-692.

3. Bouchet V, Hood DW, Li J, Brisson JR, Randle GA, Martin A, Li Z, Goldstein R, Schweda EK, Pelton Sl, et al: Host-derived sialic acid is incorporated into Haemophilus influenzae lipopolysaccharide and is a major virulence factor in experimental otitis media. Proc Natl Acad Sci USA 2003, 100(15):8898-8903.

4. Jurcisek J, Greiner L, Watanabe H, Zaleski A, Apicella MA, Bakaletz LO: Role of sialic acid and complex carbohydrate biosynthesis in biofilm formation by nontypeable Haemophilus influenzae in the chinchilla middle ear. Infect Immun 2005, 73(6):3210-3218.

5. Figueira MA, Ram S, Goldstein R, Hood DW, Moxon ER, Pelton SI: Role of complement in defense of the middle ear revealed by restoring the virulence of nontypeable Haemophilus influenzae siaB mutants. Infect Immun 2007, 75(1):325-333.

6. Swords WE, Moore ML, Godzicki L, Bukofzer G, Mitten MJ, VonCannon J: Sialylation of lipooligosaccharides promotes biofilm formation by nontypeable Haemophilus influenzae. Infect Immun 2004, 72(1):106-113.

7. Greiner LL, Watanabe H, Phillips NJ, Shao J, Morgan A, Zaleski A, Gibson BW, Apicella MA: Nontypeable Haemophilus influenzae strain 2019 produces a biofilm containing $\mathrm{N}$-acetylneuraminic acid that may mimic sialylated O-linked glycans. Infect Immun 2004, 72(7):4249-4260.

8. Vimr E, Lichtensteiger C, Steenbergen S: Sialic acid metabolism's dual function in Haemophilus influenzae. Mol Microbiol 2000, 36(5):1113-1123.

9. Vimr ER, Kalivoda KA, Deszo EL, Steenbergen SM: Diversity of microbial sialic acid metabolism. Microbiol Mol Biol Rev 2004, 68(1):132-153.

10. Severi E, Randle G, Kivlin P, Whitfield K, Young R, Moxon R, Kelly D, Hood D, Thomas GH: Sialic acid transport in Haemophilus influenzae is essential for lipopolysaccharide sialylation and serum resistance and is dependent on a novel tripartite ATP-independent periplasmic transporter. $\mathrm{Mol}$ Microbiol 2005, 58(4):1173-1185.

11. Allen S, Zaleski A, Johnston JW, Gibson BW, Apicella MA: Novel sialic acid transporter of Haemophilus influenzae. Infect Immun 2005, 73(9):5291-5300

12. Johnston JW, Zaleski A, Allen S, Mootz JM, Armbruster D, Gibson BW, Apicella MA, Munson RS Jr: Regulation of sialic acid transport and catabolism in Haemophilus influenzae. Mol Microbiol 2007, 66(1):26-39.

13. Sorensen Kl, Hove-Jensen B: Ribose catabolism of Escherichia coli: characterization of the rpiB gene encoding ribose phosphate isomerase $B$ and of the rpiR gene, which is involved in regulation of rpiB expression. J Bacteriol 1996, 178(4):1003-1011.

14. Bateman A: The SIS domain: a phosphosugar-binding domain. Trends Biochem Sci 1999, 24(3):94-95.

15. Fleischmann RD, Adams MD, White O, Clayton RA, Kirkness EF, Kerlavage AR, Bult CJ, Tomb JF, Dougherty BA, Merrick JM, et al: Wholegenome random sequencing and assembly of Haemophilus influenzae Rd. Science 1995, 269(5223):496-512.

16. Bouchet V, Huot H, Goldstein R: Molecular genetic basis of ribotyping. Clin Microbiol Rev 2008, 21(2):262-273, table of contents..

17. Cody AJ, Field D, Feil EJ, Stringer S, Deadman ME, Tsolaki AG, Gratz B, Bouchet V, Goldstein R, Hood DW, et al: High rates of recombination in otitis media isolates of non-typeable Haemophilus influenzae. Infect Genet Evol 2003, 3(1):57-66.
18. Coleman HN, Daines DA, Jarisch J, Smith AL: Chemically defined media for growth of Haemophilus influenzae strains. J Clin Microbiol 2003, 41(9):4408-4410

19. Sambrook J, Fritsch EF, Maniatis T: Molecular cloning. Cold Spring Harbor, N.Y.: Cold Spring Harbor Laboratory Press, 21989.

20. Hood DW, Deadman ME, Cox AD, Makepeace K, Martin A, Richards JC, Moxon ER: Three genes, IgtF, lic2C and IpsA, have a primary role in determining the pattern of oligosaccharide extension from the inner core of Haemophilus influenzae LPS. Microbiology 2004, 150(Pt 7):2089-2097.

21. Herriott RM, Meyer EM, Vogt M: Defined nongrowth media for stage II development of competence in Haemophilus influenzae. J Bacteriol 1970, 101(2):517-524.

22. Lesse AJ, Campagnari AA, Bittner WE, Apicella MA: Increased resolution of lipopolysaccharides and lipooligosaccharides utilizing tricine-sodium dodecyl sulfate-polyacrylamide gel electrophoresis. J Immunol Methods 1990, 126(1):109-117.

23. Pfaffl MW: A new mathematical model for relative quantification in realtime RT-PCR. Nucleic Acids Res 2001, 29(9):e45.

24. Babl FE, Pelton SI, Li Z: Experimental acute otitis media due to nontypeable Haemophilus influenzae: comparison of high and low azithromycin doses with placebo. Antimicrob Agents Chemother 2002, 46(7):2194-2199.

25. Moxon ER: Bacterial variation, virulence and vaccines. Microbiology 2009, 155(Pt 4):997-1003.

26. Schweda EK, Richards JC, Hood DW, Moxon ER: Expression and structural diversity of the lipopolysaccharide of Haemophilus influenzae: Implication in virulence. Int J Med Microbiol 2007, 297(5):297-306.

27. Trepod CM, Mott JE: Elucidation of essential and nonessential genes in the Haemophilus influenzae Rd cell wall biosynthetic pathway by targeted gene disruption. Antimicrob Agents Chemother 2005, 49(2):824-826

28. Mandrell RE, McLaughlin R, Aba Kwaik Y, Lesse A, Yamasaki R, Gibson B, Spinola SM, Apicella MA: Lipooligosaccharides (LOS) of some Haemophilus species mimic human glycosphingolipids, and some LOS are sialylated. Infect Immun 1992, 60(4):1322-1328.

29. Redfield RJ, Cameron AD, Qian Q, Hinds J, Ali TR, Kroll JS, Langford PR: A novel CRP-dependent regulon controls expression of competence genes in Haemophilus influenzae. J Mol Biol 2005, 347(4):735-747.

30. Bork P, Doolittle RF: Drosophila kelch motif is derived from a common enzyme fold. J Mol Biol 1994, 236(5):1277-1282.

31. Bauer SH, Månsson M, Hood DW, Richards JC, Moxon ER, Schweda EK: A rapid and sensitive procedure for determination of 5-N-acetyl neuraminic acid in lipopolysaccharides of Haemophilus influenzae: a survey of 24 non-typeable H. influenzae strains. Carbohydr Res 2001, 335(4):251-260.

32. Jones PA, Samuels NM, Phillips NJ, Munson RS Jr, Bozue JA, Arseneau JA, Nichols WA, Zaleski A, Gibson BW, Apicella MA: Haemophilus influenzae type $b$ strain $A 2$ has multiple sialyltransferases involved in lipooligosaccharide sialylation. J Biol Chem 2002, 277(17):14598-14611.

33. Houliston RS, Koga M, Li J, Jarrell HC, Richards JC, Vitiazeva V, Schweda EK, Yuki N, Gilbert M: A Haemophilus influenzae strain associated with Fisher syndrome expresses a novel disialylated ganglioside mimic. Biochemistry 2007, 46(27):8164-8171.

34. Steenbergen SM, Lichtensteiger CA, Caughlan R, Garfinkle J, Fuller TE, Vimr ER: Sialic Acid metabolism and systemic pasteurellosis. Infect Immun 2005, 73(3):1284-1294.

35. Severi E, Muller A, Potts JR, Leech A, Williamson D, Wilson KS, Thomas GH: Sialic Acid Mutarotation Is Catalyzed by the Escherichia coli \{beta\}Propeller Protein YjhT. J Biol Chem 2008, 283(8):4841-4849.

36. Tatum FM, Tabatabai LB, Briggs RE: Sialic acid uptake is necessary for virulence of Pasteurella multocida in turkeys. Microb Pathog 2009, 46(6):337-344.

doi:10.1186/1471-2180-10-48

Cite this article as: Jenkins et al:: Sialic acid mediated transcriptional modulation of a highly conserved sialometabolism gene cluster in Haemophilus influenzae and its effect on virulence. BMC Microbiology 2010 10:48. 